\title{
Clinical Evaluation of Moisturizers with Physiological Analysis of Stratum Corneum TARC and TSLP
}

\author{
Satoshi Fukushima ${ }^{1 *}$, Eishin Morita ${ }^{2}$, Miki Tanioka ${ }^{3}$, Yoshiki Miyachi ${ }^{3}$, Yumi Murakami ${ }^{4}$, \\ Hiroshi Matsunaka ${ }^{4}$, Hironobu Ihn ${ }^{1}$ \\ ${ }^{1}$ Department of Dermatology and Plastic Surgery, Faculty of Life Science, Kumamoto University, Kumamoto, Japan; ${ }^{2}$ Shimane \\ University Faculty of Medicine, Shimane, Japan; ${ }^{3}$ Department of Dermatology, Kyoto University Graduate School of Medicine, \\ Kyoto, Japan; ${ }^{4}$ TOKIWA Pharmaceutical Co., Ltd., Tokyo, Japan. \\ Email: "satoshi.fukushima.tb@gmail.com
}

Received December $31^{\text {st }}$, 2013; revised January $25^{\text {th }}$, 2014; accepted February $3^{\text {rd }}, 2014$

Copyright (c) 2014 Satoshi Fukushima et al. This is an open access article distributed under the Creative Commons Attribution License, which permits unrestricted use, distribution, and reproduction in any medium, provided the original work is properly cited. In accordance of the Creative Commons Attribution License all Copyrights @ 2014 are reserved for SCIRP and the owner of the intellectual property Satoshi Fukushima et al. All Copyright (C) 2014 are guarded by law and by SCIRP as a guardian.

\section{ABSTRACT}

To verify the usefulness of moisturizers, a multi-center study was conducted on patients with atopic dermatitis who visited 3 university hospitals in Japan between November 2009 and March 2012. Thirty-seven patients with dry skin and stable symptoms who were receiving topical and oral treatments were given moisturizers for 8 weeks. The moisturizers contained components such as glycerin and seawater minerals in $\mathbf{3}$ forms: lotion, emulsion, and cream. The moisturizers were chosen by physicians based on the degree of dryness of the patients' skin. In addition to observing the skin condition of the entire body, high-frequency conductance as a parameter of skin surface hydration and transepidermal water loss (TEWL) in the buccal region and the medial side of the forearm were measured, and those of the back were used for comparison. Furthermore, stratum corneum levels of thymic stromal lymphopoietin (TSLP) and thymus and activation-regulated chemokine (TARC), both of which were objective parameters for atopic dermatitis skin lesion severity, were analyzed using the tape stripping method. At the beginning of the study, TSLP showed a correlation with skin symptoms (dryness, itching) and high-frequency conductance, whereas TARC showed a correlation with skin symptoms (erythema, dryness, itching) and TEWL. In addition, a correlation was noted among TSLP, TARC, and itching. At the end of the study, erythema, dryness, itching, TEWL, TSLP, and TARC were significantly reduced, whereas high-frequency conductance was significantly increased. The moisturizers clearly improved the dry skin symptoms of these patients with atopic dermatitis and improved the physiology of their sensitive and damaged skin. These findings also support the involvement of cytokines/chemokines in the pathogenesis of atopic dermatitis lesions.

\section{KEYWORDS}

TSLP; TARC; Atopic Dermatitis; Moisturizer; Skin Physiology; Dry Skin

\section{Introduction}

In addition to its allergic aspect, atopic dermatitis involves physiological aspects of the skin, such as barrier function failure and abnormalities of the epithelium, particularly the stratum corneum [1,2]. Lipid reduction between corneocytes, such as ceramide in the stratum corneum [3]; itching related with invasion of the epidermal nerve fibers [4] and increased levels of serum TARC as

\footnotetext{
*Corresponding author.
}

indicators reflecting disease condition are well known in atopic dermatitis [5,6]. Furthermore, filaggrin gene variation has been reported in the onset of atopic dermatitis [7,8]. It is also suggested that skin care products such as moisturizers, are useful for repairing the skin barrier function as symptomatic treatment for the symptoms of atopic dermatitis, particularly dryness $[9,10]$.

In the actual treatment of atopic dermatitis in dermatology, steroids and immunosuppressive drugs are used to treat inflammation from the allergy aspect. Skin phy- 
siological abnormality due to skin barrier failure is treated with moisturizers such as Vaseline, which is used externally to prevent dryness and exacerbation of inflammation. In addition, possible prevention of the onset of atopic dermatitis by the application of moisturizers from childhood has been reported in recent years, suggesting the importance of moisturizer use [11,12]. It is also suggested that skin barrier repair using moisturizing agents is useful for preventing transdermal sensitization if the onset of atopic dermatitis is correlated with barrier function failure and its related epicutaneous sensitization.

To verify the usefulness of moisturizers in sensitive, damaged skin from the aspect of physiological function, we evaluated the external use of moisturizers in patients with atopic dermatitis and dry skin. To evaluate skin physiology, we analyzed changes in the levels of thymic stromal lymphopoietin (TSLP) and thymus and activation-regulated chemokine (TARC) in the stratum corneum [13-16], which induce allergy symptoms, promote the production of various cytokines, and are involved in the Th2 shift in addition to the generally used evaluations of high-frequency conductance as a parameter of skin surface hydration and transepidermal water loss (TEWL).

There were correlations between itching stratum corneum TSLP (scTSLP) with skin symptoms (dryness, itching) and high-frequency conductance. In addition, stratum corneum TSLP (scTARC) showed a correlation with skin symptoms (erythema, dryness, itching) and TEWL as well as with scTSLP and scTARC. In addition, improvement of the skin symptoms was related with moisturizer use. We successfully verified a significant reduction in TEWL, TSLP, and TARC; significant improvement of high-frequency conductance; and the usefulness of moisturizers from the physiological aspect.

\section{Methods}

\subsection{Subjects}

The subjects included 37 patients aged $>20$ years with atopic dermatitis, dry skin, and stabile symptoms following external and oral treatment who visited 3 university hospitals in Japan between November 2009 and March 2012. There were no serious underlying diseases. The physician in charge determined the appropriateness of the present test. Before the study was initiated, the patients were provided a thorough explanation of the study content and objectives. The subjects participated in the study of their own free will. There were no specific criteria regarding age or sex, but the subjects were only included after we had obtained written consent from them or their guardians (in case of a minor).

\subsection{Study Period and Institution}

Each subject was studied for 8 consecutive months; the study extended from November 2009 to March 2012. The institutions involved were the dermatology departments of Kumamoto University Hospital, Kyoto University Hospital, and Shimane University Hospital. The study was conducted after approval was obtained from the ethics review board of each institution.

\subsection{Study Samples}

The study samples included a lotion, emulsion, and cream marketed by TOKIWA Pharmaceutical Co., Ltd (Osaka, Japan) as treatment to improve the dry skin condition in atopic dermatitis. Three moisturizers were used for the study, all of which included Oligomarine ${ }^{\circledR}$. Oligomarine ${ }^{\circledR}$ consists of concentrated seawater and includes high concentrations of trace elements. The major ions of Oligomarine ${ }^{\circledR}$ include $\mathrm{Mg}^{2+}, \mathrm{K}^{+}, \mathrm{Zn}^{2+}, \mathrm{Se}^{4+}$, and $\mathrm{Na}^{+}$. The main moisturizing ingredients of the preparations used in this study are indicated below.

Lotion: 1, 3-butylene glycol, glycerin, hyaluronic acid.

Emulsion: glycerin, dipropylene glycol, betaine, squalane, behenyl alcohol, pentaerythrityl tetraethylhexanoate, petrolatum.

Cream: glycerin, 1, 3-butylene glycol, mineral oil, microcrystalline wax, behenyl alcohol, phytosteryl macadamiate, dipentaerythrityl tetrahydroxystearate/tetraisostearate, stearic acid, palmitic acid. These samples belong to the cosmetics category (quasi-drug) under the Japanese Pharmaceutical Affairs Law.

\subsection{Usage}

Skincare products and makeup such as cleanser and foundation used prior to the study were continued during the study. However, products with the same objective as the moisturizers were replaced with the study samples. The standard application was as follows: $2-3 \mathrm{~mL} / \mathrm{cm}^{2}$ lotion and $1-2 \mathrm{mg} / \mathrm{cm}^{2}$ emulsion or cream. The doctor in attendance decided whether the degree of dryness of the face and limbs would respond well to the lotion, emulsion, or cream. The study samples were applied twice a day: once in the morning and once in the evening (after bathing); application was adjusted according to the dry skin condition and lifestyle. One type of formulation was applied to the regions, but if the degree of dryness differed between the left and right side, we used different moisturizing agents for the two sides. The back, to which the study samples were not applied, was used for comparison.

Treatments for previously existing underlying diseases and complications were continued during the study period. In principle, the type, method, and dosage of the treatment drugs (oral and external use) were not modified during the study period; however, they were switched if the physician in charge decided that they would affect the study. Accordingly, those patients were excluded from 
the study.

\subsection{Observation and Evaluation Methods}

The physician in charge filled in a case card with each patient's information, (to avoid the possibility of connection) including identification number, age, sex, complications, and medication combination during the study period (drug, method, and dosage), with the identity of the patient concealed. The whole-body disease severity of atopic dermatitis was evaluated according to 4 stages (very mild, mild, moderate, and severe). In addition, the following items were measured and evaluated at the beginning and end of the study. Measuring points included cheek, medial side of the forearm, and back (the unapplied control). Additionally, if different formulations were used for the left and right sides, the observations were tabulated as different regions.

\subsubsection{Skin Findings}

The degree of the skin condition (erythema, dryness, and itching) of the buccal region and the medial side of the forearm, to which the study samples were applied, was scored according to 5 stages (none, 0 ; very mild, 1 ; mild, 2; moderate, 3; severe, 4).

\subsubsection{Evaluation of Skin Physiology}

After washing with soap, the measurement site was exposed to constant air conditioning (room temperature, $21^{\circ} \mathrm{C} \pm 2^{\circ} \mathrm{C}$; room humidity, $50 \% \pm 3 \%$ ) and was evaluated after $10 \mathrm{~min}$.

\section{1) High-Frequency Conductance}

High-frequency conductance, which is a parameter for the hydration state of the skin surface, was evaluated 5 times using a $3.5-\mathrm{MHz}$ high-frequency conductance measurement device (SKICON-200EX ${ }^{\circledR}$; IBS Ltd., Hamamatsu, Japan). After exclusion of the highest and lowest values, the mean of the remaining 3 values was used as the measurement value of the high-frequency conductance.

\section{2) TEWL}

TEWL was evaluated twice using a Tewameter ${ }^{\circledR} \mathrm{TM}$ 210 unit (Courage + Khazaka electronic GmbH, Cologne, Germany), and the obtained mean was used as the measurement value.

\subsubsection{Stratum Corneum TSLP and TARC Level (scTSLP and scTARC)}

Immunohistochemical staining was performed using anti-TSLP antibody (rabbit anti-human TSLP polyclonal antibody [ab47943; Abcam, Cambridge, England]) and anti-TARC antibody (mouse anti-human CCL17/TARC monoclonal antibody [MAB364; R \& D Systems, Minneapolis, MN, USA]). After staining, fluorescence images were captured using a fluorescence microscope
(BZ-8100, Keyence, Osaka, Japan), saved in Adobe ${ }^{\circledR}$ Photoshop ${ }^{\circledR}$ CS5, and the mean value of 5 visual fields of cell area luminescence was calculated as the TSLP and TARC values.

\subsubsection{Safety}

Details of adverse events (details of symptoms, degree, presence or absence of cessation, measures, and outcomes) were recorded on the case card. An event was considered a side effect if a causative correlation with the present study samples was found.

\subsection{Statistical Analysis}

Correlations among the evaluation values at the beginning of the study were analyzed using Spearman's rank correlation, whereas the changes in values between the beginning and end of the study were analyzed using a paired $t$-test or Wilcoxon's signed rank test.

\section{Results}

\subsection{Subject and Case Characteristics}

The mean age of the subjects was $27.7 \pm 10.3$ years. Whole-body disease severity was characterized as follows: very mild, 11 cases; mild, 15 cases; and moderate, 11 cases; there were no severe cases (Table 1). Complications included hay fever, asthma, allergic rhinitis, and allergy-related conjunctivitis. There were no changes to the treatment drugs (type, method, or dosage) for any

Table 1. Characterization of subjects.

\begin{tabular}{|c|c|c|c|}
\hline & & \multicolumn{2}{|c|}{ Atopic dermatitis (AD) } \\
\hline & & Male & Female \\
\hline \multirow{11}{*}{ Age } & $0-9$ & 0 & 2 \\
\hline & $10-19$ & 0 & 0 \\
\hline & $20-29$ & 13 & 10 \\
\hline & $30-39$ & 5 & 3 \\
\hline & $40-49$ & 0 & 3 \\
\hline & $50-59$ & 0 & 1 \\
\hline & $60-00$ & 0 & 0 \\
\hline & Total n & 18 & 19 \\
\hline & Mean \pm SD & $26.9 \pm 6.3$ & $28.5 \pm 13.2$ \\
\hline & Total n & \multicolumn{2}{|c|}{37} \\
\hline & Mean \pm SD & \multicolumn{2}{|c|}{$27.7 \pm 10.3$} \\
\hline \multirow{4}{*}{$\begin{array}{c}\mathrm{AD} \\
\text { Severity }\end{array}$} & Very mild & 5 & 6 \\
\hline & Mild & 4 & 11 \\
\hline & Moderate & 9 & 2 \\
\hline & Severe & 0 & 0 \\
\hline
\end{tabular}


cases during the study period.

\subsection{Skin Findings}

The scores of the skin conditions (erythema, dryness, and itching) in the buccal region and medial part of the forearm at the beginning and end of the study are listed in Table 2. At the end of the study, a significant reduction in erythema, dryness, and itching in the buccal region; dryness and itching in the medial part of the forearm; and dryness in the back were noted.

\subsection{Skin Physiology}

The high-frequency conductance and TEWL values of the buccal region, medial side of the forearm, and back at the beginning and end of the study are listed in Table 3. The significant increase in high-frequency conductance and a significant reduction in TEWL in the buccal region and medial part of the forearm were noted. The highfrequency conductance increased markedly even in the untreated back area.

\subsection{SCTSLP and TARC}

TSLP and TARC values of the buccal region, medial side of the forearm, and the back, at the beginning and end of the study are shown in Table 3. At the end of the study, there was a significant decrease in the scTSLP and scTARC levels. Stained images of the scTSLP and scTARC are shown in Figure 1.

\subsection{Safety}

None of the patients dropped out due to worsening skin condition or their own personal taste. All 37 patients successfully completed the 8-week study. All the study products were considered to be "without side effects."

\subsection{Correlation Analysis of Measurement Values}

At the beginning of the study, scTSLP showed a correlation with skin symptoms (dryness, itching) and highfrequency conductance. Additionally, scTARC was correlated with skin symptoms (erythema, dryness, itching) and TEWL as well as scTSLP and scTARC (Table 4).

\section{Discussion}

The studied skin condition was soothed with continuous treatment. We believe that the 8-week moisturizer application improved the skin conditions of the patients with atopic dermatitis and dry skin. The skin physiological tests generally used for studying the high-frequency conductance and TEWL were evaluated and exhibited improvement. In addition, to analyze the improvement of the skin condition from the physiological aspect and cellular level in detail, we evaluated TSLP and TARC in the stratum corneum.

TSLP is produced by keratinocytes during inflammation and promotes an inflammatory response related with the Th2 cytokine. This study's findings demonstrated a correlation with stratum corneum moisture levels and the improvement of dry skin after moisturizer use. TARC, a chemokine that exhibits chemotaxis properties with white blood cells, is produced in epidermal keratinocytes of lesion areas in atopic dermatitis and is involved in the local migration of lymphocytes (Th2 cells expressing CCR4), inducing IgE production, eosinophil infiltration, and activation related with the Th2-dominant immune response, and is known to express various allergic symptoms. In addition, because TSLP stimulates monocytes and produces $\mathrm{T}$ cell-suppressing chemokines such as TARC, a detailed evaluation of skin with atopic dermatitis can be achieved by analyzing local TSLP and TARC changes.

Table 2. Skin findings.

\begin{tabular}{|c|c|c|c|c|c|c|c|c|c|c|}
\hline & & \multicolumn{3}{|c|}{ Erythema score } & \multicolumn{3}{|c|}{ Dryness score } & \multicolumn{3}{|c|}{ Itching score } \\
\hline & & $\mathrm{n}$ & Mean & S.E. & $\mathrm{n}$ & Mean & S.E. & $\mathrm{n}$ & Mean & S.E. \\
\hline \multirow{3}{*}{ Cheek } & Before & 38 & 0.92 & 0.18 & 38 & 1.53 & 0.19 & 36 & 1.33 & 0.20 \\
\hline & After & 38 & 0.53 & 0.15 & 38 & 0.63 & 0.16 & 36 & 0.58 & 0.20 \\
\hline & $p$ & & $<0.01$ & $* *$ & & $<0.01$ & $* *$ & & $<0.01$ & $* *$ \\
\hline \multirow{3}{*}{ Forearm } & Before & 29 & 1.00 & 0.19 & 29 & 1.83 & 0.25 & 28 & 1.68 & 0.24 \\
\hline & After & 29 & 0.59 & 0.19 & 29 & 0.72 & 0.21 & 28 & 0.64 & 0.24 \\
\hline & $p$ & & 0.118 & N.S. & & $<0.01$ & $* *$ & & $<0.01$ & $* *$ \\
\hline \multirow{3}{*}{$\begin{array}{c}\text { Back } \\
\text { (Control) }\end{array}$} & Before & 17 & 0.412 & 0.173 & 17 & 1.294 & 0.306 & 17 & 0.76 & 0.26 \\
\hline & After & 17 & 0.235 & 0.136 & 17 & 0.824 & 0.231 & 17 & 0.53 & 0.26 \\
\hline & $p$ & & 0.083 & N.S. & & 0.038 & * & & 0.206 & N.S. \\
\hline
\end{tabular}

Wilcoxon's signed-rank test, ${ }^{* *} p<0.01,{ }^{*} p<0.05$. 
Table 3. Evaluation of skin physiology.

\begin{tabular}{|c|c|c|c|c|c|c|c|c|}
\hline & & \multirow{2}{*}{$\mathrm{n}$} & \multicolumn{2}{|c|}{ Before } & \multicolumn{2}{|c|}{ After } & \multicolumn{2}{|c|}{$p$} \\
\hline & & & Mean & S.E. & Mean & S.E. & & \\
\hline \multirow{3}{*}{ Conductance $(\mu \mathrm{S})$} & Cheek & 30 & 95.8 & 18.2 & 169.3 & 20.5 & 0.002 & ** \\
\hline & Forearm & 30 & 49.7 & 8.5 & 90.1 & 11.9 & 0.002 & ** \\
\hline & Back (Control) & 17 & 66.3 & 19.5 & 65.4 & 14.9 & 0.950 & N.S. \\
\hline \multirow{3}{*}{ TEWL $\left(\mathrm{g} / \mathrm{m}^{2} \cdot \mathrm{h}\right)$} & Cheek & 30 & 19.3 & 2.3 & 14.2 & 1.6 & 0.029 & * \\
\hline & Forearm & 30 & 19.3 & 2.9 & 12.8 & 1.8 & 0.050 & $*$ \\
\hline & Back (Control) & 17 & 20.3 & 3.3 & 16.2 & 2.8 & 0.199 & N.S. \\
\hline \multirow{3}{*}{ scTSLP level } & Cheek & 39 & 78.9 & 12.8 & 68.4 & 11.7 & 0.187 & N.S. \\
\hline & Forearm & 30 & 77.9 & 10.7 & 57.8 & 5.7 & 0.031 & * \\
\hline & Back (Control) & 14 & 26.0 & 3.3 & 21.3 & 2.6 & 0.122 & N.S. \\
\hline \multirow{3}{*}{ scTARC level } & Cheek & 39 & 59.5 & 7.7 & 46.8 & 4.5 & 0.086 & N.S. \\
\hline & Forearm & 30 & 9.5 & 1.2 & 7.8 & 1.1 & 0.017 & * \\
\hline & Back (Control) & 14 & 19.1 & 3.7 & 14.1 & 2.1 & 0.087 & N.S. \\
\hline
\end{tabular}

Paired $t$-test, ${ }^{* *} p<0.01, \stackrel{*}{p} p<0.05$.

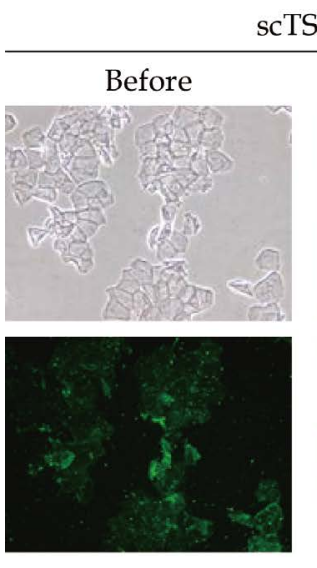

SCTSLP
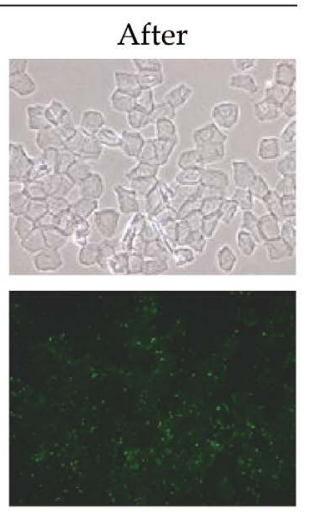

ScTARC
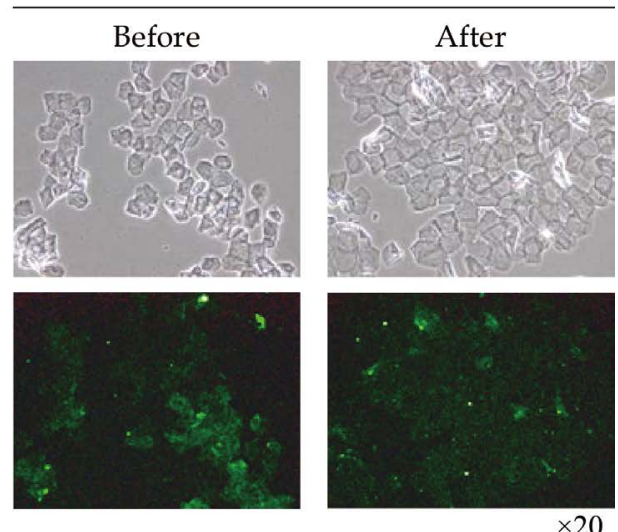

Figure 1. Immunohistochemical staining of scTSLP and scTARC.

Table 4. Correlation analysis of measurement values.

\begin{tabular}{|c|c|c|c|c|c|c|c|c|c|c|c|c|c|}
\hline \multirow{3}{*}{$\begin{array}{c}\text { scTSLP } \\
\text { level }\end{array}$} & \multirow{3}{*}{$\begin{array}{l}\mathrm{r} \\
p\end{array}$} & \multicolumn{2}{|c|}{ scTSLP level } & \multicolumn{2}{|c|}{ Conductance $(\mu S)$} & \multicolumn{2}{|c|}{ TEWL $\left(g / m^{2} \cdot h\right)$} & \multicolumn{2}{|c|}{ Erythema score } & \multicolumn{2}{|c|}{ Dryness score } & \multicolumn{2}{|c|}{ Itching score } \\
\hline & & \multicolumn{2}{|c|}{25} & \multicolumn{2}{|c|}{-0.416} & \multicolumn{2}{|c|}{0.294} & \multicolumn{2}{|c|}{0.398} & \multicolumn{2}{|c|}{0.728} & \multicolumn{2}{|c|}{0.698} \\
\hline & & & & 0.043 & * & 0.163 & N.S. & 0.060 & N.S. & $<0.01$ & $* *$ & $<0.01$ & ** \\
\hline \multirow{2}{*}{$\begin{array}{c}\text { scTARC } \\
\text { level }\end{array}$} & $\mathrm{r}$ & \multicolumn{2}{|c|}{0.632} & \multicolumn{2}{|c|}{-0.203} & \multicolumn{2}{|c|}{0.547} & \multicolumn{2}{|c|}{0.635} & \multicolumn{2}{|c|}{0.595} & \multicolumn{2}{|c|}{0.656} \\
\hline & $P$ & $<0.01$ & $* *$ & 0.342 & N.S. & $<0.01$ & $* *$ & $<0.01$ & $* *$ & $<0.01$ & $* *$ & $<0.01$ & $* *$ \\
\hline
\end{tabular}

Spearman's rank correlation, ${ }^{* *} p<0.01,{ }^{*} p<0.05$.

At the beginning of the study, scTSLP showed a correlation with skin symptoms (dryness, itching) and highfrequency conductance. In addition, scTARC showed a correlation with skin symptoms (erythema, dryness, itching) and TEWL as well with scTSLP and scTARC. This means that skin physiology and functional findings reflect the degree of skin symptoms and changes in these measurements. Following moisturizer application, reductions were seen in the skin condition score (erythema, dryness, itching), TEWL, scTSLP, and scTARC, whereas 
improved high-frequency conductance was noted. This finding suggests improvement of dry skin symptoms (stratum corneum moisture level was increased), and because decreased stimulation of epidermal keratinocytes was noted (TSLP was reduced), TARC production was controlled and the skin's inflammatory reaction was suppressed. The results also indicated improvement of the skin barrier (reduced TEWL). In the back, although no significant change with a skin physiology functional parameter was found, improvement of the shape of the dryness is accepted. The improvement of constitutional symptoms was probably caused by reduced cytokine levels. Since the subjects in the present study had skin conditions such as dry skin and inflammation prior to the moisturizer application, it is unknown whether the stratum corneum moisture level, TEWL, TSLP, or TARC primarily improved the skin symptoms. However, there was at least a close correlation between them. Thus, the improvement of dry skin conditions using moisturizers that enhance skin barrier function can be explained from the aspect of skin physiology.

\section{Conflict of Interest}

The cost and materials required were provided by TOKIWA Pharmaceutical Co., Ltd.

\section{REFERENCES}

[1] J. Callen, S. Chamlin, L. F. Eichenfield, C. Ellis, M. Girardi, M. Goldfarb, et al., "A Systematic Review of the Safety of Topical Therapies for Atopic Dermatitis,” British Journal of Dermatology, Vol. 156, No. 2, 2007, pp. 203-221.

http://dx.doi.org/10.1111/j.1365-2133.2006.07538.x

[2] D. Y. Leung and T. Bieber, “Atopic Dermatitis," The Lancet, Vol. 361, No. 9352, 2003, pp. 151-160. http://dx.doi.org/10.1016/S0140-6736(03)12193-9

[3] G. Imokawa, A. Abe, K. Jin, Y. Higaki, M. Kawashima and A. Hidano, "Decreased Level of Ceramides in Stratum Corneum of Atopic Dermatitis: An Etiologic Factor in Atopic Dry Skin?” The Journal of Investigative Dermatology, Vol. 96, No. 4, 1991, pp. 523-526. http://dx.doi.org/10.1111/1523-1747.ep12470233

[4] J. Yamaguchi, M. Aihara, Y. Kobayashi, T. Kambara and Z. Ikezawa, "Quantitative Analysis of Nerve Growth Factor (NGF) in the Atopic Dermatitis and Psoriasis Horny Layer and Effect of Treatment on NGF in Atopic Dermatitis,” Journal of Dermatological Science, Vol. 53, No. 2, 2009, pp. 48-54.

http://dx.doi.org/10.1016/j.jdermsci.2008.08.011

[5] K. Tamaki, T. Kakinuma, H. Saeki, T. Horikawa, Y. Kataoka, T. Fujisawa, et al., "Serum Levels of CCL17/ TARC in Various Skin Diseases," The Journal of Dermatology, Vol. 33, No. 4, 2006, pp. 300-302. http://dx.doi.org/10.1111/j.1346-8138.2006.00072.x

[6] T. Fujisawa, M. Nagao, Y. Hiraguchi, H. Katsumata, H.
Nishimori, K. Iguchi, et al., "Serum Measurement of Thymus and Activation-Regulated Chemokine/CCL17 in Children with Atopic Dermatitis: Elevated Normal Levels in Infancy and Age-Specific Analysis in Atopic Dermatitis,” Pediatric Allergy and Immunology, Vol. 20, No. 7, 2009, pp. 633-641. http://dx.doi.org/10.1111/j.1399-3038.2009.00851.x

[7] F. J. Smith, et al., "Loss-of-Function Mutations in the Gene Encoding Filaggrin Cause Ichthyosis Vulgaris,” Nature Genetics, Vol. 38, No. 3, 2006, pp. 337-342. http://dx.doi.org/10.1038/ng1743

[8] T. Nomura, M. Akiyama, A. Sandilands, I. Nemoto-Hasebe, K. Sakai, A. Nagasaki, T. Nomura, et al., "Prevalent and Rare Mutations in the Gene Encoding Filaggrin in Japanese Patients with Ichthyosis Vulgaris and Atopic Dermatitis," The Journal of Investigative Dermatology, Vol. 129, No. 5, 2009, pp. 1302-1305. http://dx.doi.org/10.1038/jid.2008.372

[9] M. Portugal-Cohen, M. Oron, E. Merrik, Z. Ma’or, D. Ben-Amitai, H. Yogev, et al., “A Dead Sea Water-Enriched Body Cream Improves Skin Severity Scores in Children with Atopic Dermatitis,” Journal of Cosmetics, Dermatological Sciences and Applications, Vol. 1, No. 3, 2011, pp. 71-78.

http://dx.doi.org/10.4236/jcdsa.2011.13012

[10] H. Saeki, M. Furue, F. Furukawa, M. Hide, M. Ohtsuki, I. Katayama, et al., "Guidelines for Management of Atopic Dermatitis,” The Journal of Dermatology, Vol. 36, No. 10, 2009, pp. 563-577. http://dx.doi.org/10.1111/j.1346-8138.2009.00706.x

[11] K. Kikuchi, H. Kobayashi, K. O’goshi and H. Tagami, "Impairment of Skin Barrier Function Is Not Inherent in Atopic Dermatitis Patients: A Prospective Study Conducted in Newborns,” Pediatric Dermatology, Vol. 23, No. 2, 2006, pp. 109-113.

http://dx.doi.org/10.1111/j.1525-1470.2006.00191.x

[12] E. L. Simpson, T. M. Berry, P. A. Brown and J. M. Hanifin, "A Pilot Study of Emollient Therapy For the Primary Prevention of Atopic Dermatitis," Journal of the American Academy of Dermatology, Vol. 63, No. 4, 2010, pp. 587-593. http://dx.doi.org/10.1016/j.jaad.2009.11.011

[13] J. Kawasaki, H. Ushio, H. Kinoshita, T. Fukai, F. Niyonsaba, T. Takai, et al., "Viral Infection Induces Thymic Stromal Lymphopoietin (TSLP) in Human Keratinocytes,” Journal of Dermatological Science, Vol. 62, No. 2, 2011, pp. 131-134. http://dx.doi.org/10.1016/j.jdermsci.2011.02.005

[14] Z. Zhu, M. H. Oh, J. Yu, Y. J. Liu and T. Zheng, "The Role of TSLP in IL-13-Induced Atopic March,” Scientific Reports, Vol. 1, No. 23, 2011, pp. 1-11. http://dx.doi.org/10.1038/srep00023

[15] Y. Sano, K. Masuda, R. Tamagawa-Mineoka, H. Matsunaka, Y. Murakami, R. Yamashita, et al., "Thymic Stromal Lymphopoietin Expression Is Increased in the Horny Layer of Patients with Atopic Dermatitis," Clinical \& Experimental Immunology, Vol. 171, No. 3, 2013, pp. 330-337. http://dx.doi.org/10.1111/cei.12021

[16] E. Morita, H. Takahashi, H. Niihara, I. Dekio, Y. Sumikawa, Y. Murakami, et al., "Stratum Corneum TARC 
Level Is a New Indicator of Lesional Skin Inflammation in Atopic Dermatitis,” Allergy, Vol. 65, No. 9, 2010, pp.
1166-1172.

http://dx.doi.org/10.1111/j.1398-9995.2010.02361.x

\section{Abbreviations}

TEWL: Transepidermal water loss;

TSLP: Thymic stromal lymphopoietin;

TARC: Thymus and activation-regulated chemokine;

scTSLP: Stratum corneum thymic stromal lymphopoietin; scTARC: Stratum corneum thymus and activation-regulated chemokine. 